\title{
Absorbing phase transitions of unidirectionally coupled nonequilibrium systems
}

\author{
Sungchul Kwon* and Gunter M. Schütz \\ Institut für Festkörperforschung, Forschungszentrum Jülich, D-52425 Jülich, Germany
}

(Received 11 October 2004; published 18 April 2005)

\begin{abstract}
We investigate the change of critical behavior of two-level hierarchy systems in which the second level $(B)$ is unidirectionally coupled to the first $(A)$ by the coupling dynamics $A \rightarrow A+n B$ with $n=1$ or 2 . The first level belongs to the directed percolation or the parity-conserving (PC) universality class, the second to PC. If both levels are critical, the active region of the second level becomes heterogeneous. In the so-called coupled region the first level feeds particles to the second, while in the uncoupled region the second level evolves autonomously. Measuring dynamic critical exponents in both regions, we show to what extent the critical behavior of the second level depends on the universality class of the first. These results suggest a simple criterion for the emergence of unusual critical behavior of unidirectionally coupled nonequilibrium systems.
\end{abstract}

DOI: 10.1103/PhysRevE.71.046122

PACS number(s): 64.60.-i, 05.40.-a, 82.20.-w, 05.70.Ln

\section{INTRODUCTION}

Recently there has been an enormous effort to understand nonequilibrium continuous absorbing phase transitions (APT's) from an active into absorbing states in which no further change occurs [1,2]. The concept of an APT can be applied to various phenomena in natural science as well as social and economical science. From a conceptual point of view APT's can be regarded as one of the simplest and most natural extensions of the well-established equilibrium phase transitions to nonequilibrium systems. As in equilibrium, the dimensionality of a system, symmetries between absorbing states and conservation laws may determine critical behavior of APT's. However, as a unifying theoretical framework is not available, we are still far from a systematic classification of APT's. Exact non-mean-field critical exponents and scaling functions have been derived only for systems with a singular APT where the density in the active phase diverges (see $[3,4]$ for recent progress).

Few universality classes of continuous APT's have been identified so far. The directed percolation (DP) and parityconserving (PC) universality class are well established among others [2,5]. The DP class includes systems which have no symmetry between absorbing states and no conservation laws of order parameters [1,2], while the PC class describes models with a modulo 2 conservation of the total number of particles via reactions $A \rightarrow(1+2 k) A$ and $2 A \rightarrow 0$ defining branching annihilating random walks (BAW's) with an even number of offspring [5]. The so-called directed Ising (DI) class includes models with two symmetric absorbing states [6]. In $(1+1)$ dimension the DI and PC classes are characterized by the same exponents $[2,6]$.

Unusual critical behavior has been observed in various coupled systems of known universality classes [2,7-17]. In coupled systems one (linear case) or more (nonlinear case) particles of one species give birth to an offspring of another species. However, in some cases the universality class does

*Present address: Department of Physics, Kyung Hee University, Seoul 130-701, Korea. not change. For example, quadratic and bidirectional coupling of DP processes exhibit the usual DP-type critical behavior [7]. Also linearly and bidirectionally coupled DP systems exhibit either DP or trivial critical behavior [9] depending on the existence of the spontaneous annihilation of a single particle, $A \rightarrow \varnothing[10]$. The same type of coupling of PC processes makes systems always active so only the critical behavior near the zero branching rate is observed [8]. On the other hand, cyclic coupling of DP processes and annihilating random walks may result in nontrivial critical behavior depending on a coupling constant [11]. The cyclically coupled model corresponds to the multispecies mapping of pair-contact process with diffusion [19], the universality class of which is much disputed.

Linear and unidirectional couplings have been first considered in recent studies on layer-by-layer interface growth models for adsorption and desorption of monomers [12]. It turns out that the unidirectional coupling dynamics from lower to higher layers are key features characterizing the continuously varying critical behavior of the monomer growth models $[12,14]$. Some polynuclear growth models and models for fungal growth also exhibit critical behavior of the unidirectionally coupled DP processes [15]. Using bosonic field theoretic renormalization group techniques, Täuber et al. extensively studied the hierarchy of unidirectionally coupled DP subsystems, which is a stochastic multispecies particle system $[13,14]$. Coupled PC processes have been studied in the context of interface growth models which conserve the parity of the particle number in each layer. Such dimer models undergo two different types of phase transitions, roughening and faceting transitions [17,18]. At the roughening transition, the critical behavior of the bottom layer belongs to the PC class, whereas higher layers exhibit continuously varying critical behavior.

In general, a linearly unidirectionally coupled system can be defined as a hierarchy of a number of levels belonging to known universality classes. Each level is coupled to the next level by branching dynamics of the form

$$
A_{i} \rightarrow A_{i}+n A_{i+1},
$$

with a positive integer $n$. While direct couplings with any higher levels are possible, such as $A_{1} \rightarrow A_{1}+A_{i}$, it was shown 
that they are irrelevant in determining the critical behavior $[13,14,17]$.

So far we have discussed coupled DP or PC processes. In this paper, we continue to investigate the properties of the linearly unidirectionally coupled systems in which each level can belong to a different universality class. For selfcontainedness we briefly review the usual definition of critical exponents and discuss the heterogeneity of active regions and the source average, the significance of which were detailed in recent work [16] (Sec. II). We then discuss the twolevel hierarchies. We call the first level source and the second level slave and classify the couplings between two classes as (class of source)-(class of slave) couplings according to the class of each level. In our previous study [16] we considered the cases where the source level belongs to either the DP or PC universality class and the slave level is in the DP class. Here we discuss the DP-PC (Sec. III) and PC-PC (Sec. IV) couplings, which are not investigated in [16]. In Sec. V we present simulation results of other couplings. In the concluding section VI we summarize our main findings from which we postulate a simple general criterion for the critical behavior of the slave level for general couplings.

\section{CRITICAL BEHAVIOR AND AVERAGING IN UNIDIRECTIONALLY COUPLED SYSTEMS}

\section{A. Critical exponents in coupled APT's}

As in the usual APT's, several critical exponents characterize the off-critical and critical behavior of the coupled systems. Near criticality the exponents $\beta, \beta^{\prime}, \nu_{\perp}$, and $\nu_{\|}$ characterize the scaling behavior of the steady-state particle density $\rho_{s}$, the survival probability $P_{s}$, the correlation length $\xi$, and the characteristic time $\tau$ in the thermodynamic limit as [20]

$$
\rho_{s} \sim \Delta^{\beta}, \quad P_{s} \sim \Delta^{\beta^{\prime}}, \quad \xi \sim|\Delta|^{-\nu_{\perp},} \quad \tau \sim|\Delta|^{-\nu_{\|}},
$$

where $\Delta$ is the distance from criticality.

At criticality the exponent $\alpha$ characterizes the decay of the density of particles $\rho(t)$ in the surviving samples when starting with random initial conditions with finite density, while for a single-particle initial condition the exponents $\delta$, $\eta$, and $z$ characterize the survival probability $P_{s}(t)$ of the processes, the number of particles $N(t)$ averaged over all samples, and spreading distance $R(t)$ averaged over survival samples, respectively, as [21]

$$
\rho(t) \sim t^{-\alpha}, \quad P_{s}(t) \sim t^{-\delta}, \quad R(t) \sim t^{1 / z}, \quad N(t) \sim t^{\eta} .
$$

Dynamical scaling implies the relations $\alpha=\beta / \nu_{\|}, \delta$ $=\beta^{\prime} / \nu_{\|}, z=\nu_{\|} / \nu_{\perp}$. The well-known scaling relation between these exponents in the usual APT is [21]

$$
\eta=d / z-\alpha-\delta .
$$

In many systems $\beta$ and $\beta^{\prime}$ are equal [21] and hence $\alpha=\delta$.

The critical behavior where criticality of all levels coincides is characterized by a varying order parameter exponent $\beta$ taking a different value in each level of the hierarchy. Within the mean-field approximation, the exponent $\beta$ of a level $k$ is simply given by $\beta_{k}=(1 / 2)^{k}$ with $k \geqslant 0$ for both the coupled DP and PC processes $[13,14]$. This $\beta_{k}$ obtains corrections below the upper critical dimension $\left(d^{C}\right)$.

On the other hand, the exponents $\nu_{\perp}$ and $\nu_{\|}$remain unchanged in all levels, but $\delta$ and $\eta$ vary according to the level. However these exponents do not satisfy the ordinary scaling relation Eq. (4). Instead for the coupled DP processes they are suggested to satisfy the following relation in each level $k$ [14]:

$$
\eta_{k}=d / z_{k}-\alpha_{k}-\delta_{1},
$$

with $\alpha_{k}=\delta_{k}$. The exponent $\delta_{1}$ of $P_{s}(t)$ of the first level replaces $\delta_{k}$ of the level $k$. Generally $\alpha_{k}$ is the exponent for the particle density at level $k$ for random initial conditions at the first level. In what follows we refer to the first level as source and higher levels as slave levels.

\section{B. Heterogeneous spreading and source average}

The derivation of Eq. (5) from scaling arguments of [14] involves some tacit assumptions on taking averages which may lead to wrong conclusions if ignored. It has been shown that for the generalization of Eq. (5) to other couplings and for understanding the appearance of $\delta_{1}$, it is necessary to take the heterogeneity of active regions on the slave levels into account and to apply a particular average method, the source average [16]. It turns out that the active regions of the slave levels are divided into two regions, the so-called coupled and uncoupled region. Careful analysis yields generalized scaling relations consisting of two separate equations for the two regions respectively [16]. For self-containedness we briefly review the significance of these notions.

\section{Source average}

By construction of the process, i.e., due to the feeding of particles to higher levels, each level decays more slowly than its lower levels. So the coupling to lower levels is always broken after some time. From this time on the decoupled level $k$ evolves autonomously according to its own dynamics, with critical exponents given by the pure (uncoupled) universality class to which this level belongs. Therefore a trivial change of universality class at level $k$ occurs abruptly when the lower level $k-1$ dies out. Thus no information about different universality classes due to coupling can be gained by studying realizations of the process with broken coupling.

In order to avoid this problem we only consider the configurations in which the source $(k=1)$ still survives. In these configurations, slave levels $(k>1)$ always survive with unit probability due to the coupling so the survival probability $P_{s}(t)$ of the slave is just that of the source and we have the following relation for any $k>1$ :

$$
\delta_{k} \neq \alpha_{k}, \quad \delta_{k}=\delta_{1} .
$$

We call these configurations the source ensemble and the average with this ensemble we refer to as the source average. Of course, one may average dynamic quantities over the configurations in which the level under consideration survives regardless of the survival of other levels. This implies $\delta_{k}$ 


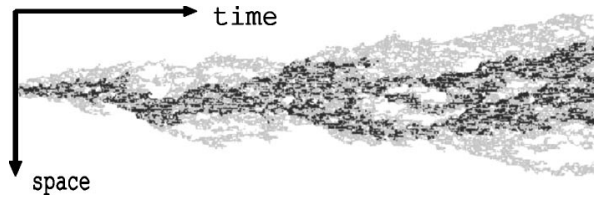

FIG. 1. The evolution pattern of a cluster on the second level starting from a single particle on the first level. The gray regions outside the black regions are uncoupled.

$\neq \delta_{1}$ for general couplings. We refer to this average as the slave average, but we shall not employ it for the reasons given above.

Our discussion not only explains the occurrence of $\delta_{1}$ in Eq. (5) for coupled DP systems but also suggests that $\delta_{1}$ will occur for any coupling, defined via the source average. The source average more clearly exhibits the special properties of unidirectionally coupled systems than the usual slave average and it allows the derivation of the scaling relations without any assumptions on scaling functions for observables.

\section{Dynamic heterogeneity}

The origin of the dynamic heterogeneity of the slave levels can be understood as follows. Starting with a single particle on the source level $(k=1)$, then in level $k(>1)$ a cluster of particles $\left(A_{k}\right)$ is formed by the coupling dynamics (1) and it spreads according to the given evolution rules. Due to the coupling, the average size of the cluster on each level is always larger than those of the lower levels. It means that the cluster of the level $k$ with size $R_{k}(t)$ is divided into two parts, the coupled and the uncoupled region. The coupled region of the level $k$ at time $t$ is defined as the set of all sites within the range between the leftmost and the rightmost particles of the level $k-1$ at time $t$. The exterior of the coupled region is the uncoupled region of the level $k$ (Fig. 1).

Inside the uncoupled region with size $R^{U}(t)=R_{k}(t)$ $-R_{k-1}(t)$, the dynamics on the level $k$ evolve autonomously without particle feeding from the level $k-1$. In the coupled region with size $R^{C}(t)$, the level $k-1$ feeds particles to the level $k$ so that $R^{C}(t)$ is actually the spreading distance $R_{k-1}(t)$ of the level $k-1$. Due to the coupling, the number of particles $N^{C}(t)$ inside the coupled region increases more rapidly than that of the uncoupled region $\left[N^{U}(t)\right]$. This observation suggests that the number of particles in the level $k$ can increase in time with different exponents, $\eta_{k}^{C}$ in the coupled region and $\eta_{k}^{U}$ in the uncoupled region respectively, at the multicritical point,

$$
N^{C}(t) \sim t^{\eta_{k}^{C}}, \quad N^{U}(t) \sim t^{\eta_{k}^{U}}
$$

We stress that even though inside the uncoupled region the particle system evolves according to the critical dynamics of level $k$, the critical exponents may be different. This is because the boundaries of the uncoupled region (and hence its overall size and therefore its critical exponents) are determined by the coupling to the lower level. It is clear that this heterogeneity of the particle distribution of the level $k\left[N_{k}(t)\right]$ is a general property of unidirectionally coupled systems.

\section{Generalized scaling relations}

We discuss the hyperscaling in the level $k$ in $d$ dimensions, using the source average. The index $k$ is omitted from the number of particle $N$ and density $\rho$ in the coupled and uncoupled region for simplicity of notation. In the coupled region, $N^{C}(t)$ is given by the relation

$$
N^{C}(t)=\rho^{C}(t) \times\left(R^{C}\right)^{d}(t) \times P_{s}^{k=1}(t),
$$

where $\rho^{C}(t)$ is the particle density of the level $k$ of surviving samples inside of the volume $\left(R^{C}\right)^{d}(t)$ and $P_{s}^{k=1}(t)$ is the survival probability of the source level. Assuming algebraic decay at the multicritical point $\rho^{C} \sim t^{-\alpha_{k}^{C}}, R^{C}=R_{k-1} \sim t^{1 / z_{k-1}}$, and $P_{s}^{k=1} \sim t^{-\delta_{1}}$, respectively, we obtain the first scaling relation

$$
\eta_{k}^{C}=d / z_{k-1}-\alpha_{k}^{C}-\delta_{1} .
$$

In the uncoupled region, assuming $\rho^{U}(t) \sim t^{-\alpha_{k}^{U}}$ and $R^{U}$ $\sim t^{1 / z_{k}^{U}}$, we find the second relation

$$
\eta_{k}^{U}=d / z_{k}^{U}-\alpha_{k}^{U}-\delta_{1} .
$$

The above two relations are generalized scaling relations for unidirectionally coupled systems taking the heterogeneity into account. They are very different from Eqs. (4) and (5) because they are not scaling relations for quantities of the whole system. As $N_{k}(t)$ is given by $N_{k}(t)=N_{k}^{C}(t)+N_{k}^{U}(t)$, the scaling of $N_{k}(t)$ is determined by the scaling behavior of the two distinct regions

$$
\eta_{k}=\max \left[\eta_{k}^{C}, \eta_{k}^{U}\right] .
$$

For the scaling of the total size of the level $k R_{k}=R_{k}^{U}+R_{k-1}$ $\sim t^{1 / z_{k}}$, we have the condition, $z_{k}^{U} \geqslant z_{k}$, or

$$
1 / z_{k}=\max \left[1 / z_{k}^{U}, 1 / z_{k-1}\right] .
$$

Similarly one has for the total density $\rho_{k}(t) \sim t^{-\alpha_{k}}$ the relation

$$
\alpha_{k}=\min \left[\alpha_{k}^{C}, \alpha_{k}^{U}\right] .
$$

Notice that for the special case of $z_{k}^{U}=z_{k-1}$, the exponent $\eta_{k}^{C}$ is larger than $\eta_{k}^{U}$ because $\alpha_{k}^{C}$ and $\alpha_{k}^{U}$ satisfy the condition $\alpha_{k}^{C} \leqslant \alpha_{k}^{U}$. Therefore, we have two equalities of $\eta_{k}=\eta_{k}^{C}$ and $\alpha_{k}=\alpha_{k}^{C}$ and we recover the scaling relation of Eq. (5) but with $\alpha_{k} \neq \delta_{k}$. In general, as dynamic exponents such as $\eta_{k}$ and $\delta_{k}$ depend on the average methods, the value of $\eta_{k}$ of the slave average differs from that of the source average.

\section{Corrections to scaling}

We remark that Eqs. (9) and (10) also quantify the corrections to scaling of dynamic quantities coming from the generic heterogeneity of coupled systems. The effective exponent of $N_{k}(t)$ is defined as

$$
\eta_{k}(t)=\ln \left[N_{k}(m t) / N_{k}(t)\right] / \ln m,
$$

and similarly for $\rho_{k}(t)$ and $R_{k}(t)$ with a positive $m$. The heterogeneity induces corrections to scaling of the form $t^{-\Delta_{\rho}}$, $t^{-\Delta_{\eta}}$, and $t^{-\Delta_{z}}$ with some negative exponents 


$$
\Delta_{\rho}=\left|\alpha_{k}^{C}-\alpha_{k}^{U}\right|, \quad \Delta_{\eta}=\left|\eta_{k}^{C}-\eta_{k}^{U}\right|, \quad \Delta_{z}=\left|1 / z_{k}^{U}-1 / z_{k-1}\right| .
$$

These correction exponents usually become very small and therefore they cause a significant long time drift of the effective exponents. These corrections explain the difficulties of precise estimations of the dynamic exponents in unidirectionally coupled systems.

\section{DP-PC COUPLING}

To simplify notation for two-level couplings we call particles of the source $(k=1)$ and the slave level $(k=2) A$ and $B$, respectively. Then for the slave level we have the scaling relations from Eqs. (9) and (10)

$$
\begin{aligned}
& \eta^{C}=1 / z_{A}-\alpha^{C}-\delta_{A}, \\
& \eta^{U}=1 / z^{U}-\alpha^{U}-\delta_{A},
\end{aligned}
$$

where we omit the index $B$ from $\eta, \alpha$, and $z$. Relations (11)-(13) become

$$
\begin{gathered}
\eta=\max \left[\eta^{C}, \eta^{U}\right], \\
1 / z=\max \left[1 / z^{U}, 1 / z_{A}\right], \\
\alpha=\min \left[\alpha^{C}, \alpha^{U}\right] .
\end{gathered}
$$

For DP-PC coupling, we consider the contact process (CP) and branching annihilating random walks with two offspring $[\mathrm{BAW}(2)]$ as typical particle models belonging to the DP and PC class, respectively [22,23]. In the CP of the source level, a particle $A$ is spontaneously annihilated with rate $p$ and it creates one $A$ particle on one of the nearest neighboring $(\mathrm{NN})$ sites with rate $(1-\sigma)(1-p)$. In branching processes, if the target site is occupied by an other particle, the branching is rejected. The rate $\sigma$ is the coupling strength. The criticality is $p_{c}^{A}=0.232674(4)$ at $\sigma=0$ [22]. Using the fact that the ratio of the creation and annihilation rates, $R$ $=\left(1-p_{c}\right) / p_{c}$, should be same at criticality for any value of $\sigma$, we find that the critical line of CPs in the $\sigma-p$ phase diagram is $\sigma_{c}^{A}=1-p R_{A} /(1-p)$ with $R_{A}=3.29785$.

In the BAW(2) of the slave level, a particle $B$ hops to one of the NN sites with rate $p$ and it creates two particles on two NN sites to the left or right direction with $\sigma(1-p)$. If two particles happen to be on a same site by branching or hopping, they annihilate each other instantaneously. The critical point of BAW(2) at $\sigma=1$ is located at $p_{c}^{B}=0.5105(7)$ [23]. The critical line is $\sigma_{c}^{B}=p R_{B} /(1-p)$, where $R_{B}=\left(1-p_{c}^{B}\right) / p_{c}^{B}$ $=0.95886$.

The coupling dynamics of Eq. (1) now becomes

$$
A \rightarrow A+n B \quad \text { with } \sigma(1-p),
$$

where we take without loss of generality $n=2$.

The coupling dynamics (20) couples the $\mathrm{CP}$ and $\mathrm{BAW}(2)$ without feedback from the BAW(2) to the CP. An $A$ particle creates $B$ particles on neighboring sites of the same site of the BAW level only if the target sites are empty. The multi-

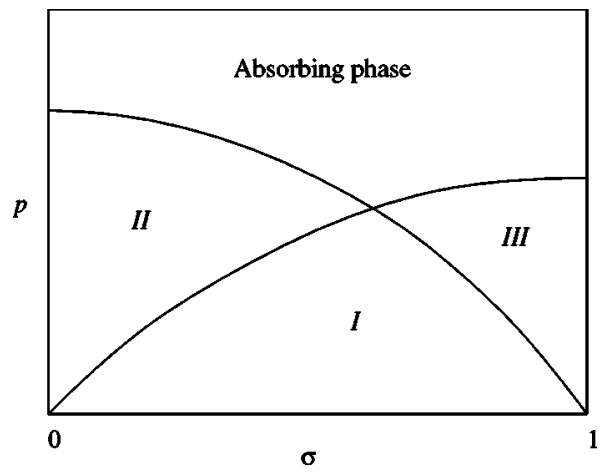

FIG. 2. Schematic phase diagram of two-level hierarchies with coupling strength $\sigma$ and interaction parameter $p$. The region labeled by "Absorbing phase" corresponds to an absorbing phase of both levels.

critical point $\left(\sigma_{M}, p_{M}\right)$ of the CP-BAW hierarchy is located at $\sigma_{M}=0.22526$ and $p_{M}=0.19023$ within errors of $p_{c}^{A}$ and $p_{c}^{B}$. The well-known values of the exponents $\delta$ and $2 / z$ of the source level are $\delta_{A} \approx 0.159$ and $2 / z_{A} \approx 1.265$ [2].

Figure 2 shows the schematic general phase diagram of two-level hierarchies. In region I, the two levels are both in an active phase of nonzero steady-state particle density. In region II, both levels are active but the slave level is slaved to the first. The slave level undergoes the same type of phase transition as the source level at the critical line of the source level. In region III, two levels are completely decoupled. While the source level is inactive, the slave level is active. They undergo their own types of phase transition at their respective critical points.

First we perform dynamic Monte Carlo simulations starting with a single $A$ particle on the source level at the multicritical point $\left(\sigma_{M}, p_{M}\right)$. For the average of dynamic quantities we take the source average. Using the effective exponents of Eq. (14), we obtain asymptotic values of the exponents $\eta, \alpha$, and $1 / z$ of the slave level in the coupled and uncoupled regions, respectively. We list all measured values of the exponents for all couplings in Tables I and II.

Figure 3 shows effective exponents as functions of time of the coupled (uncoupled) region and the whole active region. The different values of the exponents in the both regions clearly show the heterogeneity of the growing cluster generated from a single $A$ particle. By substituting the measured values of $\eta^{C}$ and $\eta^{U}$ into Eq. (16), we predict $\alpha^{C}$ $=0.133(10)$ and $\alpha^{U}=0.30(2)$ which agree very well with the

TABLE I. Measured values of critical exponents of the slave level for the four couplings. The subscript $C(U)$ corresponds to the coupled (uncoupled) region. $\eta_{\text {pred }}^{C}$ and $\eta_{\text {pred }}^{U}$ are the predicted values from Eq. (16) using measured values.

\begin{tabular}{ccccccc}
\hline \hline Coupling & $\alpha^{C}$ & $\alpha^{U}$ & $\eta^{C}$ & $\eta^{U}$ & $\eta_{\text {pred }}^{C}$ & $\eta_{\text {pred }}^{U}$ \\
\hline DP-PC & $0.13(1)$ & $0.28(1)$ & $0.34(1)$ & $0.14(1)$ & $0.34(1)$ & $0.16(2)$ \\
DP-DP & $0.08(1)$ & $0.16(1)$ & $0.40(1)$ & $0.31(1)$ & $0.39(1)$ & $0.31(2)$ \\
PC-DP & $0.14(2)$ & $0.16(1)$ & $0.14(2)$ & $0.185(5)$ & $0.15(2)$ & $0.185(20)$ \\
PC-PC & $0.21(1)$ & $0.285(5)$ & $0.08(1)$ & $0.01(1)$ & $0.08(1)$ & $0.00(1)$ \\
\hline \hline
\end{tabular}


TABLE II. Measured values of $2 / z_{B}$ and $2 / z^{U}$ for the four couplings. The values of $2 / z_{B \text {,pred }}$ are estimated values from Eq. (18).

\begin{tabular}{ccccc}
\hline \hline $2 / z$ & DP-PC & DP-DP & PC-DP & PC-PC \\
\hline $2 / z^{U}$ & $1.20(2)$ & $1.27(2)$ & $1.26(2)$ & $1.15(1)$ \\
$2 / z_{B}$ & $1.24(1)$ & $1.27(2)$ & $1.22(2)$ & $1.15(1)$ \\
$2 / z_{B, \text { pred }}$ & 1.265 & $1.27(2)$ & 1.265 & $1.15(1)$ \\
\hline \hline
\end{tabular}

numerical estimates of Table I within errors. All dynamic exponents satisfy the generalized relations of (16) well.

To obtain exponents of the total quantities $\rho_{B}, N_{B}$ and $R_{B}$, we should compare the values of the coupled and uncoupled region according to Eqs. (17)-(19). As $N^{C}$ increases more rapidly than $N^{U}\left(\eta^{C}>\eta^{U}\right), N_{B}$ is expected to follow the scaling of $N^{C}$. It implies $\eta_{B}=\eta^{U}$. Similarly $\rho_{B}$ and $R_{B}$ should follow the scaling of $\rho^{C}$ and $R^{C}\left(=R_{A}\right)$ due to $\alpha^{C}<\alpha^{U}$ and $1 / z^{C}>1 / z^{U}$ respectively. So we expect $\alpha_{B}=\alpha^{C}$ and $z_{B}=z_{A}$ for DP-PC coupling. However in Fig. 3, $\eta_{B}(t)$ saturates to a smaller value than $\eta^{C}$. The other exponents $\alpha_{B}(t)$ and $2 / z_{B}(t)$ also show the same tendency. As mentioned in Sec. II, these discrepancies come from the corrections to scaling with the exponents defined in Eq. (15). Directly measuring the effective slope of the correction to $N_{B}$, viz., through the ratio $N_{B}(t) / N^{C}(t)$, we obtain $\Delta_{\eta}=0.20(1)$ which agrees with the prediction $\Delta_{\eta}=\eta^{C}-\eta^{U}=0.20(2)$. Similarly we also find correction exponents $\Delta_{\alpha}=0.15(1)$ and $\Delta_{z}=0.03(1)$, which agree

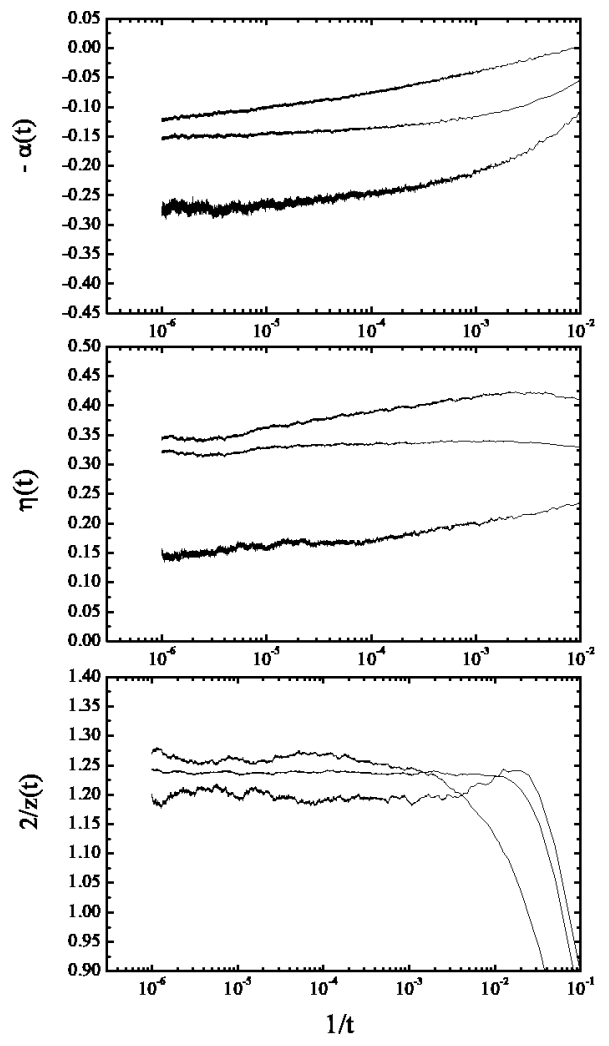

FIG. 3. Effective exponents $\alpha, \eta, 2 / z$ of the slave level for DP-PC coupling. From top to bottom in each panel, each line corresponds to the coupled, whole, and uncoupled regions, respectively.

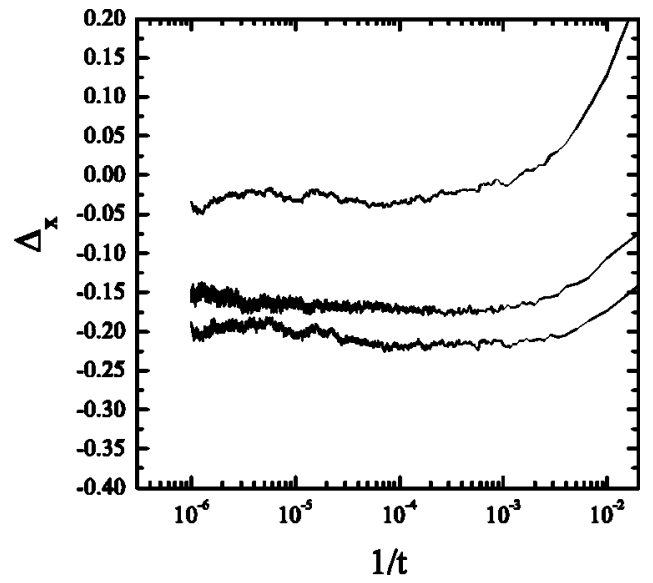

FIG. 4. Effective exponents of corrections to scalings of the slave level for DP-PC coupling. From top to bottom, each line corresponds to $\Delta_{z}, \Delta_{\eta}$, and $\Delta_{\alpha}$.

well with the predictions $\Delta_{\alpha}=0.15(1)$ and $\Delta_{z}=0.02(2)$, respectively. Figure 4 shows the effective exponents of the ratios. We should note that the corrections come from the heterogeneity of a growing cluster. The effect of coupling causes the long drift of effective exponents as shown in $\alpha^{C}(t)$ especially.

Since the dynamic exponents $\eta$ and $\delta$ generally depend on the average methods [6], the dynamic critical behavior should be determined by $\alpha$ and $z$. The exponents $\alpha$ and $z$ defined in Eq. (3) are the ratios of static exponents $\beta, \nu_{\perp}$, and $\nu_{\|}$in an active phase where all levels in the hierarchy survive with finite probability. The finite survival probability of each level implies the equivalence of source and slave average so the static exponents are independent of average methods.

From this analysis we see that due to coupling with a DP process the spreading properties of the PC process BAW(2) completely change and show DP nature. It can be understood in general terms from the rapid spreading of DP processes with smaller $z$ than that of the PC class $\left(z^{U}>z^{C}\right)$. As the typical sizes of DP vacuum domains $\left(\xi_{\perp}\right.$ and $\left.\xi_{\|}\right)$in an active phase are smaller than those of the PC class, vacuum domains of PC class shrink by the activation of the source level and they should follow the scaling of the DP class. It implies that $\nu_{\perp}$ and $\nu_{\|}$should be the values of the DP class so we have $z=z_{\mathrm{DP}}$. So the DP-PC coupling completely changes the scaling of both spatial and temporal correlation lengths, unlike other couplings.

On the other hand, as the DP processes on the source level survive longer than PC processes do, the coupling with the source makes the decay of the total density $\left(\rho_{B}\right)$ slow enough to change the exponent $\alpha$ in the coupled region $\left(\alpha^{C}\right)$. The $\alpha^{C}$ of Table I is even smaller than the value of the DP class, $\alpha_{\mathrm{DP}} \approx 0.16$. It is consistent with the fact that the exponent $\beta$ changes and actually decreases as levels go up as in other unidirectionally coupled systems. With the DP value of $\nu_{\|}$, we predict $\beta_{B}=\alpha^{C} \nu_{\|}^{D P}=0.23(1)$.

For the measurement of the exponent $\beta$ of the slave level, we measure $\rho_{B}$ in the steady state $\left(\rho_{B}^{s}\right)$ with half-filled random initial conditions on the source level. We average $\rho_{B}^{s}$ for $\Delta=p_{M}-p=8 \times 10^{-2}-2 \times 10^{-4}$ and system sizes $L=4096-1.2$ 


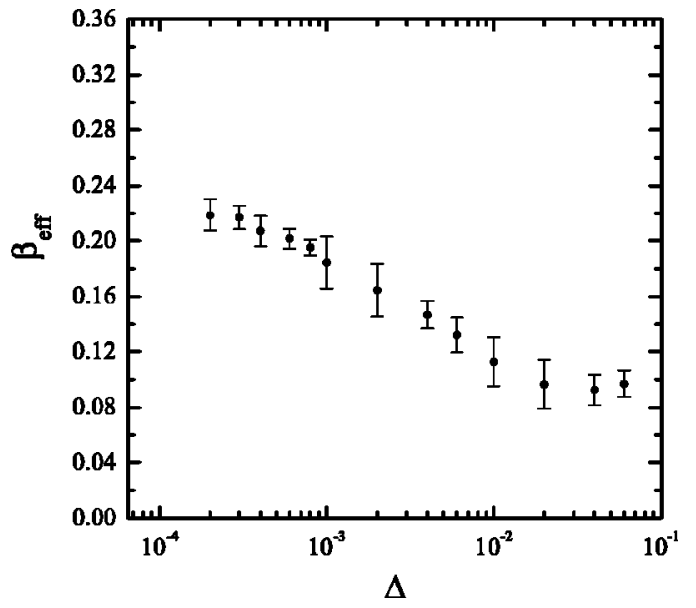

FIG. 5. Effective exponents $\beta_{\text {eff }}$ of $\rho_{B}^{s}$ for DP-PC coupling.

$\times 10^{4}$ (Fig. 5). We find $\beta_{B}=0.22(2)$ which agrees very well with our prediction. We conclude that while the coupling between the same type processes only changes the exponent $\beta$ of higher levels, DP-PC coupling completely changes the scaling behavior of the spatial and temporal correlation length in addition to $\beta$ of the slave level.

\section{PC-PC COUPLING}

In PC-PC coupling, we then consider BAW(2) model in both levels. Although the coupling was already studied [17], we investigate the critical behavior in a more detailed and transparent manner using the heterogeneity and source average.

In the source level, an $A$ particle hops with rate $p$ and creates two offsprings on two nearest neighbor sites to the left or to the right with rate $(1-\sigma)(1-p)$. In the slave level, hopping and branching of a $B$ particle occur with rates $p$ and $\sigma(1-p)$, respectively. The two levels are unidirectionally coupled with the coupling dynamics Eq. (20). The critical lines of the two levels are $\sigma_{c}^{A}=1-p R /(1-p)$ and $\sigma_{c}^{B}$ $=p R /(1-p)$ with $R=0.95886$. The multicritical point is located at $\sigma_{M}=1 / 2$ and $p_{M}=0.342732$. The well-known exponents $\delta$ and $2 / z$ of the source level are $\delta_{A}=0.285(2)$ and $2 / z_{A}=1.141(2)$ [24].

Starting with two $A$ particles on the source level, we measure the effective exponents $\alpha, \eta$, and $1 / z$ in the coupled and uncoupled regions (Fig. 6). We performed simulations for $n=1$ and 2 . The two results are very similar so we present results only for the more natural case $n=2$ (Tables I and II).

As shown in Table I, the particle number of the coupled region $\left(N^{C}\right)$ increases more rapidly so that the total particle number $N_{B}$ follows the scaling of $N^{C}$ and we expect $\eta_{B}$ $=\eta^{C}$. Similarly we expect $\alpha_{B}=\alpha^{C}$ for the total particle density. Figure 6 shows a small deviation between the total and the coupled regions, but it can be explained by the corrections to scaling. From Eq. (15) we estimate correction exponents $\Delta_{\alpha}=0.185(10)$, and $\Delta_{\eta}=0.07(1)$.

With the measured values of $\eta^{C}, 1 / z^{U}$ given in the table and $\delta_{A}$ and $z_{A}$ of the PC class, we predict $\alpha^{C}=0.21(1)$ and $\alpha^{U}=0.28(1)$ which agree well with the numerical results in
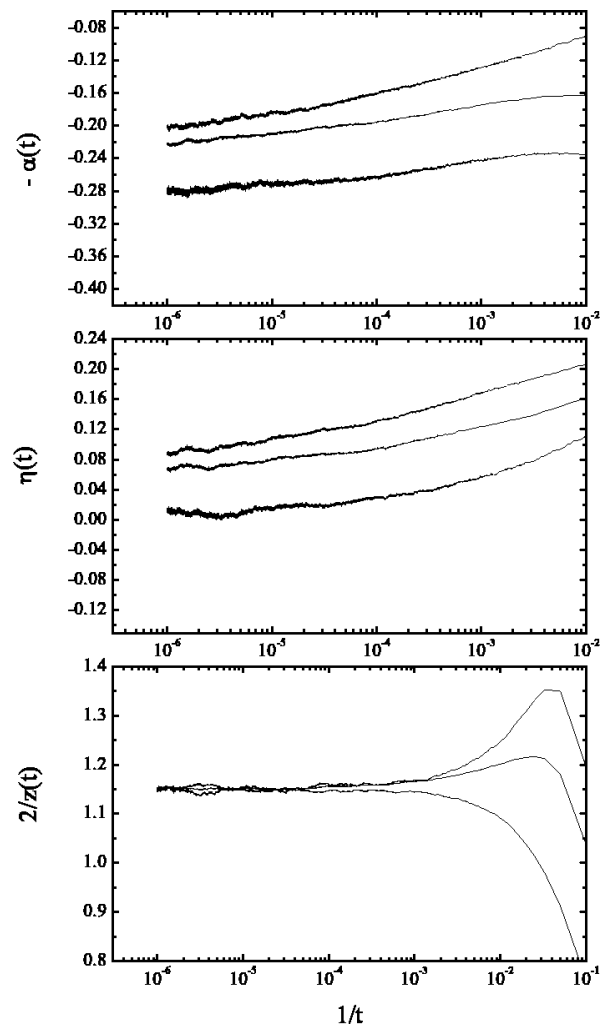

FIG. 6. Effective exponents of the slave level for PC-PC coupling. From top to bottom in each panel, each line corresponds to the coupled, whole, and uncoupled regions respectively.

Table I. Hence Eq. (16) is satisfied very well in PC-PC coupling. The PC-PC coupling only changes $\eta^{C}$ and $\alpha^{C}$, like the DP-DP coupling. As the spatial and temporal correlation lengths follow the same scaling in both levels before the coupling, the exponents $\nu_{\perp}$ and $\nu_{\|}$of both levels should be the same after the coupling. This leads to $z_{A}=z_{B}$. Only the density in the coupled region decays more slowly than in the PC class. This implies the decrease of $\beta$ in the slave level. We estimate $\beta_{B}=0.68(1)$ with the relation $\beta_{B}=\alpha^{C} \nu_{\|}^{P C}$, which is smaller than $\beta_{P C}=0.92(3)$ [24].

\section{OTHER COUPLINGS}

In this section we present some improved simulation results on DP-DP and PC-DP couplings, respectively, and we briefly illustrate the essential importance of using the source average for the validity of hyperscaling relations for the three-level PC-DP-DP couping. For DP-DP coupling, we consider two CP models. In PC-DP coupling, $A$ particles of $\mathrm{BAW}(2)$ evolve on the source level with hopping rate $p$ and two-offspring branching rate $(1-\sigma)(1-p)$. In both couplings, the two levels are coupled through the dynamics (20). For the location of the corresponding critical points see [16].

In DP-DP coupling, effective exponents of the coupled region saturate to asymptotic values as shown in Fig. 7, which also well satisfy the scaling relation Eq. (16). The same value of $z$ in both coupled and uncoupled regions indicates that the scaling of spatial and temporal correlation 


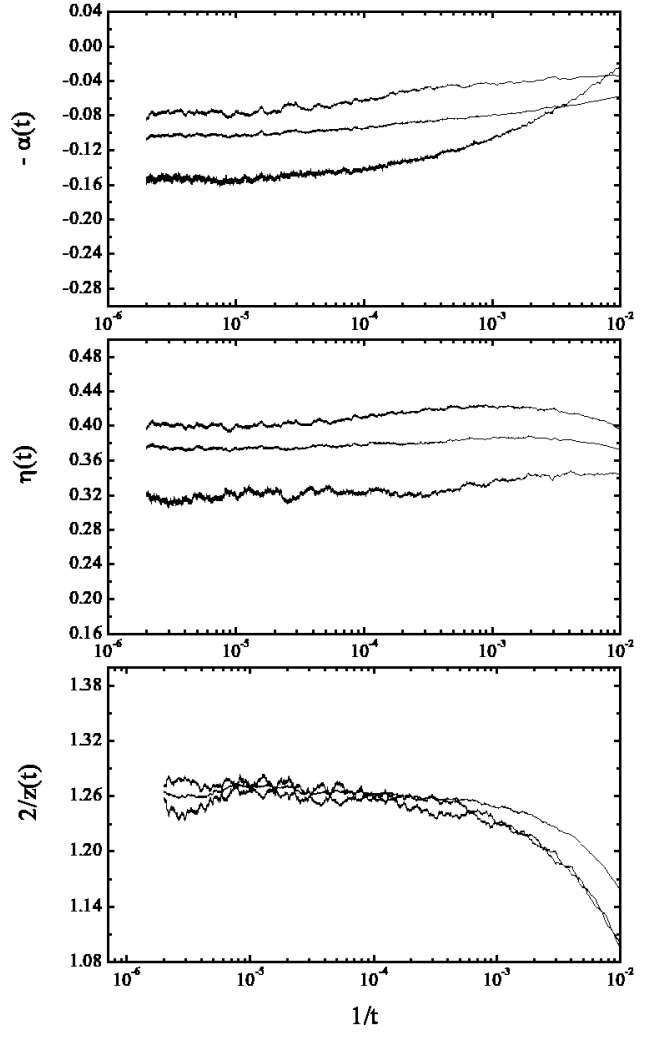

FIG. 7. Effective exponents of the slave level for DP-DP coupling. From top to bottom in each panel, each line corresponds to the coupled, whole, and uncoupled regions, respectively.

length remains unchanged as noted in Refs. $[13,14]$. However, the small value of $\alpha^{C}$ indicates the decrease of $\beta$. With the relation $\beta=\alpha^{C} \nu_{\|}^{\mathrm{DP}}$ we estimate $\beta=0.14(1)$ which is compatible with the result of [14], $\beta_{2}=0.132(15)$.

In PC-DP coupling, the DP level is expected to show DPtype critical behavior because $\mathrm{PC}$ processes decay faster with $\alpha_{\mathrm{PC}}>\alpha_{\mathrm{DP}}$. The fast decay and slow spreading of PC processes yields for the exponent of total particle number $\eta_{B}$ $=\eta^{U}$ rather than $\eta^{C}$, unlike in other couplings. Assuming DP critical behavior, we expect $\eta^{C}=0.13, \eta^{U}=0.185, \alpha^{C}=\alpha^{U}$ $=0.16$, and $2 / z_{B}=1.265$. This is well borne out by the simulation results, even though Fig. 8 shows that the exponents of the coupled region converge to the expected values very slowly. The long drift of $\alpha^{C}$ and $\eta^{C}$ comes from the effect of the coupling dynamics by itself, while the total spreading distance $R_{B}(t)$ has strong corrections to scaling $R^{C} / R^{U}$ $\sim t^{-\Delta_{z}}$.

With the values of $\eta$ and $z$ in Tables I and II, Eq. (16) predicts $\alpha^{C}=0.15(2)$ and $\alpha^{U}=0.16(2)$, which agree with the $\mathrm{DP}$ value $\alpha_{\mathrm{DP}}=0.16$ [2]. The scaling relation of Eq. (16) is well satisfied for PC-DP coupling although the particle density decays uniformly in the whole region of the slave level. On the other hand, with the slave average, all exponents including $\eta_{B}$ should be DP values, which satisfy the ordinary scaling relation of Eq. (4) rather than Eqs. (5) and (16). As Eq. (5) is the result of Eq. (16), we conclude that the slave average actually cannot satisfy the scaling relations of unidirectionally coupled systems. However, the results of a slave average satisfy Eq. (5) in other couplings. This contradiction
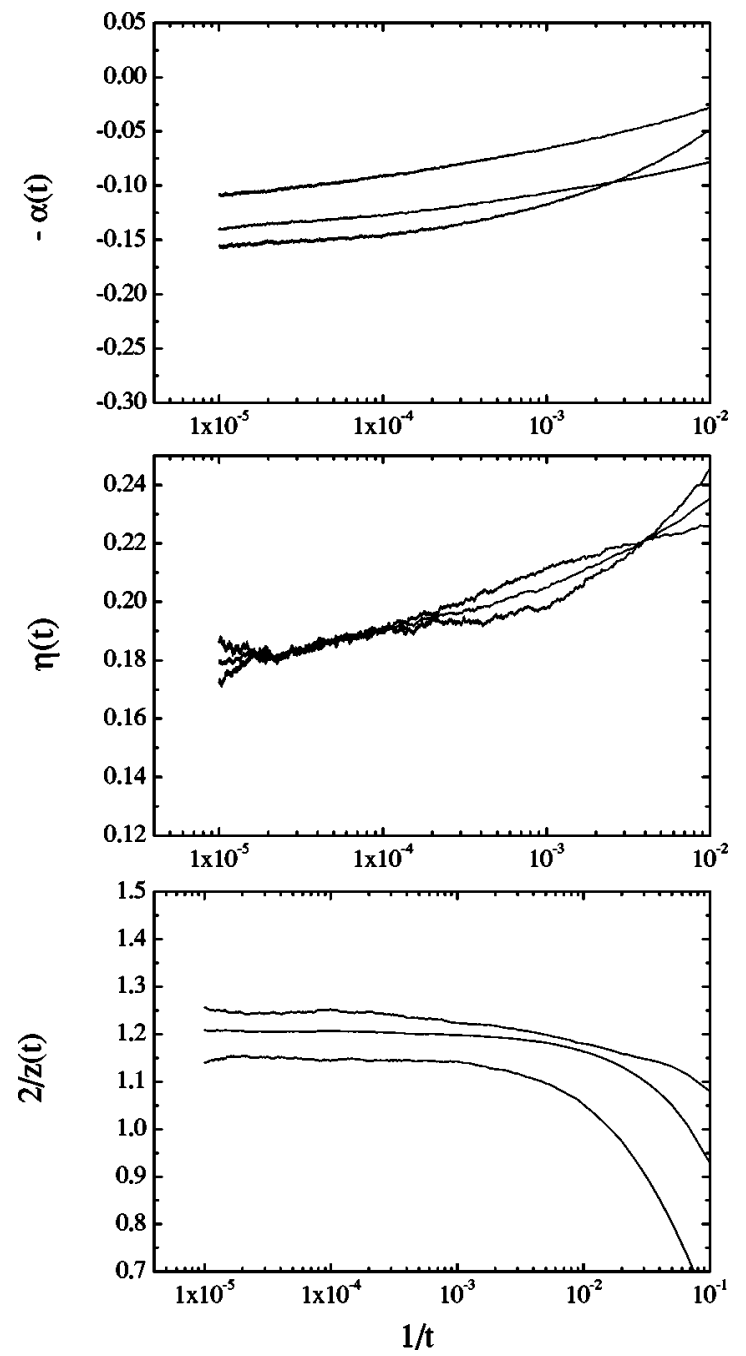

FIG. 8. Effective exponents of the slave level for PC-DP coupling. From bottom to top in each panel, each line corresponds to the coupled, whole, and uncoupled regions, respectively. $\eta(t)$ shows differences only after $5 \times 10^{4}$ time steps.

can be explained by examining the composition of the slave ensemble. In the slave average, as we only consider the configurations in which the slave level under consideration survives, the source level may survive or already have disappeared. So the ensemble of the slave average includes some configurations of source average which characterize the critical behavior of higher levels. This is why results of the slave average can satisfy Eq. (5).

The three-level hierarchy of PC-DP-DP coupling is another example where the average method is crucial for the validity of hyperscaling relations. In this hierarchy, the critical behavior of the third level is affected by the second, but the second is not affected by the first. So if we use the slave average, the exponents of the third level should be those of the slave level of DP-DP coupling. These exponents of the slave level satisfy only the scaling relation of the two-level hierarchy without the PC process in which $\delta_{1}$ is the DP value. However, as $\delta_{1}$ in the scaling relation of the threelevel hierarchy is the $\mathrm{PC}$ value, the exponents of slave average cannot satisfy Eq. (5) of the PC-DP-DP coupling. 


\section{CONCLUSION}

In summary, measuring static and dynamic exponents, we investigate the change of critical behavior of two-level systems in which the slave level is unidirectionally coupled to the source level through the coupling dynamics $A \rightarrow A+n B$. Each level belongs to either the DP or PC universality class. The coupling makes the active region of the slave level inhomogeneous. Therefore the particle number exhibits different scalings in two regions, the so-called coupled and uncoupled regions. Proper analysis of the change of scaling properties due to the coupling necessitates the use of the source average. The heterogeneity of the particle distribution in the slave level is characteristic for all unidirectionally coupled systems and leads to generalized scaling relations which are valid irrespective of whether the critical behavior of higher levels is changed or not by given couplings.

However, it should be noted that the slave average may be better than the source average in other regions of the phase diagram Fig. 2. This comes from the fact that except at the multicritical point, the slave levels are completely uniform and we cannot divide the whole active region into two distinct regions. For example, in region III of the generic phase diagram, the two levels are completely decoupled and the source cannot affect the critical behavior of the slave because the source is inactive. So we cannot define the coupled region which is the characteristic of unidirectionally coupled systems at their common critical point. In region II, the slave level is completely slaved to the source so the whole active region of the slave level is the coupled region. In this region we cannot define the uncoupled region and we have just one scaling relation of the coupled region.
At the multicritical point the critical behavior strongly depends on that of the source level. For DP-DP and PC-PC couplings, the coupling only changes the scaling of the order parameter. The scalings of spatial and temporal correlation lengths do not change. For DP-PC coupling, the DP level completely changes the critical behavior of the PC level. While the spatial and temporal correlation lengths follow the scaling of the DP class, the order parameter exhibits slower decay. However, in PC-DP coupling, the PC level is too weak to change the critical behavior of the DP level.

These results for the four couplings suggest the following simple criterion for the critical behavior of the slave level in unidirectionally coupled two-level hierarchies.

(1) The coupling of models belonging to the same class only changes the scaling of the order parameter as already noted in $[13,14,17]$.

(2) The coupling of more slowly with more quickly spreading systems such as DP-PC coupling completely changes the critical behavior of the slave level. While the spatial and temporal correlation lengths follow the scaling of the source level, the order parameter decays more slowly with a smaller exponent $\beta_{B}$ than that of the source.

(3) Finally, the coupling of faster decaying with more slowly spreading systems such as PC-DP coupling does not change the critical behavior of the slave. The coupling only makes the slave converge to its asymptotic critical behavior very slowly and it causes a long drift of effective exponents.

Since in unidirectionally coupled systems of more than two levels each level is affected by the level below, it is possible to apply the above criterion of two-level hierarchies to general hierarchies.
[1] J. Marro and R. Dickman, Nonequilibrium Phase Transitions in Lattice Models (Cambridge University Press, Cambridge, U.K., 1999).

[2] H. Hinrichsen, Adv. Phys. 49, 815 (2000).

[3] B. Houchmandzadeh, Phys. Rev. E 66, 052902 (2002).

[4] M. Paessens and G. M. Schütz, J. Phys. A 37, 4709 (2004).

[5] J. Kockelkoren and H. Chaté, Phys. Rev. Lett. 90, 125701 (2003).

[6] W. Hwang, S. Kwon, H. Park, and H. Park, Phys. Rev. E 57, 6438 (1998).

[7] H. K. Janssen, Phys. Rev. Lett. 78, 2890 (1997).

[8] J. L. Cardy and U. Täuber, Phys. Rev. Lett. 77, 4780 (1996); S. Kwon, J. Lee, and H. Park, ibid. 85, 1682 (2000).

[9] S. Kwon and H. Park, J. Korean Phys. Soc. 38, 490 (2001); G. Ódor, Phys. Rev. E 63, 021113 (2001).

[10] S. Kwon and H. Park, Phys. Rev. E 69, 066125 (2004).

[11] H. Hinrichsen, Physica A 291, 275 (2001).

[12] U. Alon, M. R. Evans, H. Hinrichsen, and D. Mukamel, Phys. Rev. Lett. 76, 2746 (1996).
[13] U. C. Täuber, M. J. Howard, and H. Hinrichsen, Phys. Rev. Lett. 80, 2165 (1998).

[14] Y. Y. Goldschmidt, H. Hinrichsen, M. J. Howard, and U. C. Täuber, Phys. Rev. E 59, 6381 (1999).

[15] A. Toom, J. Stat. Phys. 74, 91 (1994); J. M. López and H. J. Jensen, Phys. Rev. Lett. 81, 1734 (1998).

[16] S. Kwon and G. M. Schütz, Physica A 341, 136 (2004).

[17] H. Hinrichsen and G. Ódor, Phys. Rev. Lett. 82, 1205 (1999).

[18] J. D. Noh, H. Park, and N. den Nijs, Phys. Rev. Lett. 84, 3891 (2000).

[19] M. Henkel and H. Hinrichsen, J. Phys. A 37, R117 (2004).

[20] T. Aukrust, D. A. Browne, and I. Webman, Phys. Rev. A 41, 5294 (1990).

[21] P. Grassberger and A. de la Torre, Ann. Phys. (N.Y.) 122, 373 (1979).

[22] T. E. Harris, Ann. Prob. 76, 1122 (1982); I. Jensen and R. Dickman, J. Stat. Phys. 79, 89 (1993).

[23] S. Kwon and H. Park, Phys. Rev. E 52, 5955 (1995).

[24] I. Jensen, Phys. Rev. E 50, 3623 (1994). 